\title{
Preparing and Characterization of Some Heterocyclic Compounds with Studying Their Biological Activity
}

\author{
Muhanned A. Mahmoud \\ Department of Chemistry, College of Sciences for Women, University of Baghdad. \\ E-mail: alnemimu2006@yahoo.com.
}

\begin{abstract}
New compounds of (E-(1,3-bis)3-bromophenyl) prop-2-en-1-one], (E-(1-(3-Nitrophenyl-(3-(1h-pyrrol-2-yl) prop-2-en-1-one], (E-(1-(3-Nitrophenyl-(3-(1H-pyrrol-2-yl) prop-2-en-1-one], [(E)N,N-1,3-bis) 3-Nitrophenyl) propan-3-yl-1-ylidene) dimethan amine], (E-(1-(3-Nitrophenyl-(3-(1H-pyrrol-2-yl) prop-2-en-1-one] and [(E-(1,3-bis)4-dimethylamino) phenyl) prop-2-en-1-one] have been synthesized. The prepared compounds have synthesized from chalcone [1] and characterized by using FT-IR, Uv/ vis and ${ }^{1} \mathrm{H}-\mathrm{NMR}$ spectra besides, determining the melting points and Rf with the biological activity.
\end{abstract}

Keywords: Heterocyclic compounds, pyrrols, chalcones.

\section{Introduction}

Chalcones are well known intermediates for synthesizing various heterocyclic compounds. The compounds with the backbone of chalcones have been reported to possess various biological activities such as antimicrobial $^{[1-3]}$, anti-inflam - matory $^{[4]}$, antimalaria $^{[5-6]}$, antilies-hmanial ${ }^{[7],}$ antioxidant $^{[8]}$ andantitubercular $^{[9]}$.

Chalcones are an aromatic ketone and an enone that forms the central core for a variety of important biological compounds, which are known collectively as chalcones. Aldol condensation represent an important class of carbon-double bond carbon formation reactions both in nature and in synthetic chemistry. Compounds called chalcones ${ }^{[10]}$ can prepare by aldol condensation of an aromatic ketone and aldehyde.

Pyrazole also, is one of a class of organic heterocyclic compounds containing a five member aromatic ring structure composed of two nitrogen atoms and three carbon. But pyrazoline it is a class of organic heterocyclic compounds containing a five member not aromatic ring structure composed of two nitrogen atoms and three carbon ${ }^{[11]}$. In the present work we report the reaction of various substituted acetophenone with different substituted aromatic aldehyde to form chalcones ${ }^{[12]}$ and then converted to pyrolles.

\section{Experimental}

Melting points were recorded with Stuart Melting point apparatus and were uncorrected. Infra red spectra (FT-IR) were recorded on Shimadzu FT-IR-8300 spectrophotometer in Ibn Sina State Company (ISSC).Uv/vis spectra were recorded on Uv/vis varian Uv-Cary-100 spectrophoto-meters in (ISSC).1H-NMR spectra were recorded on a BRUKER-400 $\mathrm{MHz}$ operating at $300 \mathrm{MHZ}$ with tetra methyl silane as internal standard in $\mathrm{CDCl}_{3}$ and DMSO-d6 as a solvent, measurements were made at Chemistry Department, AL-Baath University-Syria. Elemental Analysis (C.H.N.S.) was carried out with: Euroea Elemental Analyzer Italia by Chemical Department College of Science, Babylon University. Thin layer Chromatography (TLC) was carried out by using alumina plates percolated with silica-gel, supplied by Merck. Spots were detected with iodine vapor. The biological activity was performed by Biology Department College of Science, University of Tikrit.

1) Synthesis of 3- (2-substitutedphenyl) -1(-3-nitrophenyl) prop-2-en-1-one (1-5)

To a stirred mixture of 3-nitro acetophenone $(0.01 \mathrm{~mol})$ and substituted benzaldehyde $(0.01 \mathrm{~mol})$ in absolute ethanol $(5 \mathrm{ml})$ and $\mathrm{NaOH}$ in ethanol $(30 \%)$ and continue stirring for two hours at room temperature. Allow to stand reaction mixture for 12 hours. Precipitate the reaction mixture 
by addition of water, acidified with diluted $\mathrm{HCl}$. Filter the product, wash with cold ethanol and allowed to afford.

\section{2-Synthesis of (3-phenyl-4, 5-dihydro-1H-} pyrazol - 5-yl)benzene (6-10).

Chalcone (1-5) $(0.01 \mathrm{~mol})$ was dissolved in ethyl alcohol 95\% (20ml) and refluxed with excess ofhydrazine hydrate for $12 \mathrm{hrs}$, the reaction mixture was diluted with cold water $50 \mathrm{ml}$ and the white precipitate formed was filtered off and recrystallized with ethyl alcohol.

\section{Results and Discussion}

This paper reports a simple and effective method for the synthesis of chalocnes by an basic catalyzed aldol reaction we used $\mathrm{NaOH}$ as a convenient method. Chalcones are obtained in good to excellent yields. Our purpose was to synthesize a series of chalcones, starting from benzaldehyde and acetophenone or their substituted derivatives.

Synthesis of chalcone is a single step method. The synthesized chalcone derivatives were undergone physicochemical character ization and the obtained results are given in (Table (2)). The yields of the synthesized compounds were found to be significant. The structure of the synthesized compounds was confirmed by IR, ${ }^{1} \mathrm{H}-\mathrm{NMR}$, Uv/vis spectra.

All the compounds give the characteristic IR band that proved the presence of particular functional group (Table (2)) and ${ }^{1} \mathrm{H}-\mathrm{NMR}$, Uv/vis spectroscopy helps to find the molecular weight structures of the synthesized compounds (Table (3)). The IR band at $1778 \mathrm{~cm}^{-1}$ suggesting the presence of $(\mathrm{C}=\mathrm{O})$ group, at $1631 \mathrm{~cm}^{-1}$ due to $(\mathrm{C}=\mathrm{C})$ group (compound 1). The IR band at $1606 \mathrm{~cm}^{-1}$ indicates the presence of $(\mathrm{C}=\mathrm{C})$ group. FT-IR band at $3142 \mathrm{~cm}^{-1}$ indicates the presence of $(-\mathrm{OH})$ group (compound (2) as example). TLC, melting points, FT-IR spectroscopy for compounds (6-10) proved the presence of $(\mathrm{C}=\mathrm{N})$ group and $(\mathrm{N}-\mathrm{H})$. Also, the FT-IR band at $1591 \mathrm{~cm}^{-1}$ indicates the presence of $(\mathrm{C}=\mathrm{C})$ group. FT-IR band at $3261 \mathrm{~cm}^{-1}$ indicates the presence of $(-\mathrm{OH})$ group. The results obtained from this study confirmed that the product has formed. Henceforth viewing these characteristic properties more compounds can be synthesized and subjected to pharmacological evaluation.

UV spectrum Fig.(3) shows the transions $\mathrm{n} \rightarrow \pi$ and $л \rightarrow \Omega^{*}$ which confirmed the presence of the un-bonded pair of electrons on nitrogen atom and aromatic system (double bond). The product (1) is also, identifiedby the $1 \mathrm{H}$ NMRspectrum which shows the protons at $(\delta$ 7.5-8) ppm due to aromatic protons. Proton of $(\mathrm{N}-\mathrm{H})$ of pyrazole ring appeared at $\delta(8.05)$ Fig.(2).

The FT-IR spectrum of compound (10), shows the bands, Fig. (4), at $\left(3250 \mathrm{~cm}^{-1}\right)$, $\left(2924\right.$ and $\left.2854 \mathrm{~cm}^{-1}\right)$ are attributed to $v(\mathrm{C}-\mathrm{H})$ aromatic, and (C-H) aliphatic stretching vibrations of $(\mathrm{C}-\mathrm{H})$ group. Other characteristic bands of aromatic system is the appearance of $v(\mathrm{C}=\mathrm{C})$ at about $(1512 \mathrm{~cm}-1)$ besides the band at $(1640 \mathrm{~cm}-1)$ due to $(\mathrm{C}=\mathrm{N})$.

The 1H-NMR spectrum of compound [10], shows the following characteristic chemical shifts (DMSO-d6) ppm. Protons of $(\mathrm{CH} 2)$ of pyrazole ring appeared at $(\delta 4.22)$. Proton of $(\mathrm{NH})$ group appeared at $(\delta 4.5)$. Protons of aromatic rings appeared at the range $\delta(7.5-8)$ as a multiplate peaks.

Table (1)

Physical properties of compounds [1-10].

\begin{tabular}{|c||c||c||c||c||c|}
\hline $\begin{array}{c}\text { Comp. } \\
\text { No. }\end{array}$ & $\begin{array}{c}\text { Substitute } \\
\text { d groups }\end{array}$ & $\begin{array}{c}\text { Molecular } \\
\text { formula }\end{array}$ & $\begin{array}{c}\text { Molecular } \\
\text { weight }\end{array}$ & $\begin{array}{c}\text { M.P/ } \\
\boldsymbol{C}\end{array}$ & $\begin{array}{c}\text { Yield } \\
\%\end{array}$ \\
\hline \hline 1 & $\mathrm{NO}_{2}$ & $\mathrm{C}_{15} \mathrm{H}_{10} \mathrm{~N}_{2} \mathrm{O}_{5}$ & 298 & $\begin{array}{c}120- \\
122\end{array}$ & 80 \\
\hline \hline 2 & $\mathrm{OH}$ & $\mathrm{C}_{15} \mathrm{H}_{11} \mathrm{NO}_{4}$ & 269 & $\begin{array}{c}135- \\
137\end{array}$ & 69 \\
\hline 3 & $\mathrm{Br}$ & $\mathrm{C}_{15} \mathrm{H}_{10} \mathrm{NO}_{3} \mathrm{Br}$ & 332 & $\begin{array}{c}144- \\
147\end{array}$ & 77 \\
\hline 4 & $\mathrm{~N}-\mathrm{Me}$ & $\mathrm{C}_{17} \mathrm{H}_{16} \mathrm{~N}_{2} \mathrm{O}_{3}$ & 296 & $\begin{array}{c}141- \\
143\end{array}$ & 73 \\
\hline \hline 5 & $\mathrm{Pyrrole}$ & $\mathrm{C}_{13} \mathrm{H}_{10} \mathrm{~N}_{2} \mathrm{O}_{3}$ & 242 & $\begin{array}{c}125- \\
127\end{array}$ & 70 \\
\hline \hline 6 & $\mathrm{NO}$ & $\mathrm{C}_{15} \mathrm{H}_{12} \mathrm{~N}_{4} \mathrm{O}_{4}$ & 312 & $\begin{array}{c}156- \\
158\end{array}$ & 78 \\
\hline \hline 7 & $\mathrm{OH}$ & $\mathrm{C}_{15} \mathrm{H}_{13} \mathrm{~N}_{3} \mathrm{O}_{3}$ & 283 & $\begin{array}{c}175- \\
177\end{array}$ & 62 \\
\hline \hline 8 & $\mathrm{Br}$ & $\mathrm{C}_{15} \mathrm{H}_{12} \mathrm{~N}_{3} \mathrm{O}_{2}$ & 346 & $\begin{array}{c}170- \\
173\end{array}$ & 74 \\
\hline \hline 9 & $\mathrm{~N}-\mathrm{Me}$ & $\mathrm{C}_{17} \mathrm{H}_{18} \mathrm{~N}_{4} \mathrm{O}_{2}$ & 310 & $\begin{array}{c}180- \\
182\end{array}$ & 69 \\
\hline \hline 10 & pyrrole & $\mathrm{C}_{13} \mathrm{H}_{12} \mathrm{~N}_{4} \mathrm{O}_{2}$ & 256 & $\begin{array}{c}162- \\
164\end{array}$ & 85 \\
\hline \hline
\end{tabular}


Table (2)

The C.H.N. analysis for some prepared compounds.

\begin{tabular}{||c||c||c||c||c||}
\hline $\begin{array}{c}\text { Comp. } \\
\text { No. }\end{array}$ & M.F. & C\% & H\% & N\% \\
\hline \hline \multirow{2}{*}{4} & \multirow{2}{*}{$\mathrm{C}_{17} \mathrm{H}_{16} \mathrm{~N}_{2} \mathrm{O}_{3}$} & Cal.68.91 & 5.40 & 9.45 \\
\cline { 3 - 5 } & & Found68.92 & 5.43 & 9.42 \\
\hline \hline 7 & $\mathrm{C}_{15} \mathrm{H}_{13} \mathrm{~N}_{3} \mathrm{O}_{3}$ & Cal.63.60 & 4.59 & 14.84 \\
\cline { 3 - 5 } & Found63.58 & 4.55 & 14.90 \\
\hline \hline 9 & $\mathrm{C}_{17} \mathrm{H}_{18} \mathrm{~N}_{4} \mathrm{O}_{2}$ & Cal. 65.80 & 5.80 & 18.06 \\
\cline { 3 - 5 } & Found. 65.77 & 5.86 & 18.02 \\
\hline \hline 10 & $\mathrm{C}_{13} \mathrm{H}_{12} \mathrm{~N}_{4} \mathrm{O}_{2}$ & Cal.60.93 & 4.68 & 21.87 \\
\cline { 3 - 5 } & Found60.89 & 4.62 & 21.93 \\
\hline
\end{tabular}

Table (3)

The Rf of the prepared compounds.

\begin{tabular}{|c||c||c|}
\hline Comp. No. & $\boldsymbol{R}_{\boldsymbol{f}}$ & Solvent \\
\hline \hline 1 & 0.92 & Dioxane \\
\hline \hline 2 & 0.84 & $=$ \\
\hline \hline 6 & 088 & $=$ \\
\hline \hline 1 & 076 & $=$ \\
\hline \hline 10 & 0.81 & $=$ \\
\hline
\end{tabular}

Table ( $\varepsilon)$

FT-IR spectral data of compounds [1-5].

\begin{tabular}{|c|c|c|c|c|c|c|}
\hline $\begin{array}{l}\dot{8} \\
\dot{\vec{g}} \\
\dot{0}\end{array}$ & : & 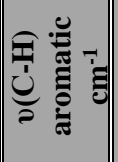 & 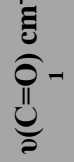 & 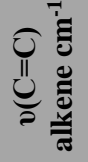 & 导产 & 离 \\
\hline- & 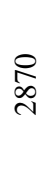 & $\begin{array}{l}\text { Oి } \\
\stackrel{\infty}{\infty}\end{array}$ & $\stackrel{\infty}{\Sigma}$ & $\overline{\widehat{B}}$ & 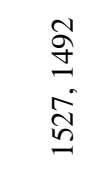 & 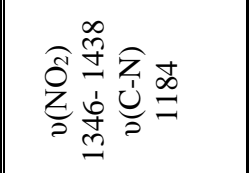 \\
\hline$N$ & 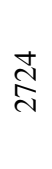 & 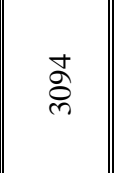 & $\stackrel{\cong}{\beth}$ & $\widehat{\widehat{్}}$ & 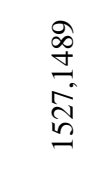 & 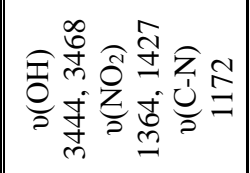 \\
\hline$m$ & $\stackrel{\curvearrowright}{\stackrel{\sim}{\sim}}$ & 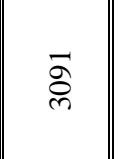 & 气ิ & $\stackrel{\curvearrowright}{\widehat{\sigma}}$ & 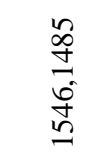 & 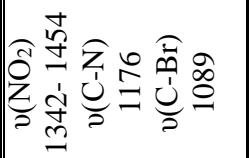 \\
\hline 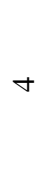 & 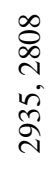 & ô & $\stackrel{0}{I}$ & f & 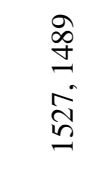 & 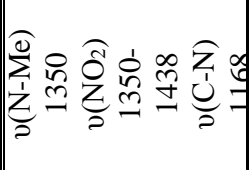 \\
\hline in & 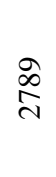 & ồ & $\begin{array}{l}\stackrel{\text { I }}{\text { I }} \\
\text { ¿ }\end{array}$ & $\stackrel{0}{6}$ & 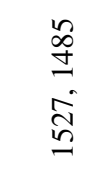 & 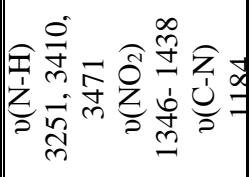 \\
\hline
\end{tabular}

Table ( $\left.{ }^{\bullet}\right)$

FT-IR spectral data of compounds [6-10].

\begin{tabular}{|c|c|c|c|c|c|c|}
\hline $\begin{array}{l}\dot{\dot{z}} \\
\dot{\vec{\Xi}} \\
\dot{0}\end{array}$ & 䄈 & 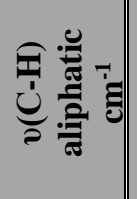 & |气 & 坖 & 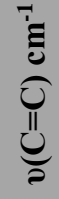 & 离 \\
\hline 0 & స్ & 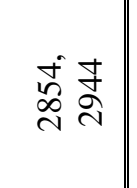 & हे & $\underline{\sigma}$ & $\begin{array}{l}\infty \\
\stackrel{n}{ \pm} \\
\vec{n} \\
\stackrel{n}{n}\end{array}$ & 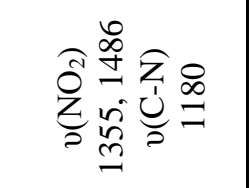 \\
\hline$r$ & $\begin{array}{l}\infty \\
\stackrel{\sim}{\sim}\end{array}$ & 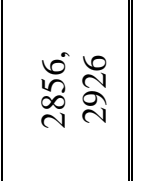 & ڤેे & $\stackrel{8}{0}$ & $\begin{array}{l}\hat{\delta} \\
+ \\
\infty \\
0 \\
0 \\
n\end{array}$ & 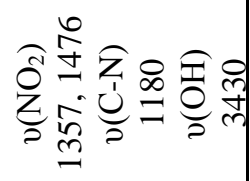 \\
\hline$\infty$ & 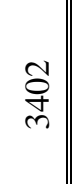 & 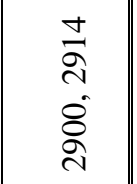 & ఓి & $\begin{array}{l}\text { हे } \\
\text { an }\end{array}$ & $\begin{array}{l}0 \\
\text { o } \\
0 \\
\hat{n} \\
n\end{array}$ & 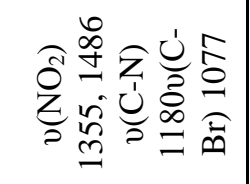 \\
\hline$a$ & $\begin{array}{c}\infty \\
\text { } \\
\text { లె }\end{array}$ & $\begin{array}{l}\frac{n}{\grave{\lambda}} \\
\frac{\widehat{त}}{a ̀}\end{array}$ & 令 & $\frac{0}{6}$ & 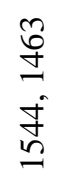 & 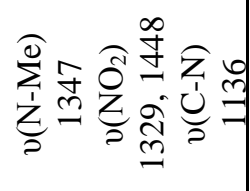 \\
\hline 으 & 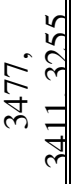 & 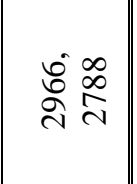 & 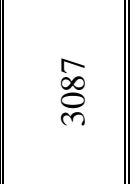 & $\begin{array}{l}\mathscr{1} \\
\underline{\sigma}\end{array}$ & $\begin{array}{l}\infty \\
\stackrel{\infty}{ \pm} \\
\stackrel{i}{n}\end{array}$ & 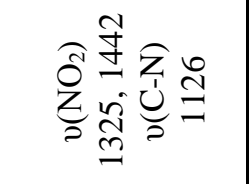 \\
\hline
\end{tabular}

\section{Microbiological Method}

In this work, the antibacterial test was performed according to thedisc diffusion method. Compounds ((2), (4), (6), (8), (10)) were assayed for their antimicrobial activity in vitro against Gram-negative bacteria (Escherichia coli) and Gram-positive bacteria (staphylococcus aureus). Prepared agar and Petridishes were sterilized by autoclaving for $15 \mathrm{~min}$ at $121 \mathrm{C}^{\circ}$. The agar plates were surface inoculated uniformly from the broth culture of the tested microorganisms. In the solidified medium suitably spaced apart holes were made all $6 \mathrm{~mm}$ in diameter. These holes were filled with $100 \mu \mathrm{l}$ of the prepared compounds $(1 \mathrm{mg}$ of the compound dissolved in $1 \mathrm{ml}$ of DMSO solvent), DMSO was used as a solvent. These plates were incubated at $37 \mathrm{C}^{\circ}$ for $24 \mathrm{~h}$ for both bacteria. The inhibition zones caused by the various compounds were examined. The results of the preliminary screening tests are listed in Table ( 7 ). 
Table (

Antibacterial activities of some of the synthesized compounds.

\begin{tabular}{|c||c||c|}
\hline $\begin{array}{c}\text { Comp. } \\
\text { No. }\end{array}$ & $\begin{array}{c}\text { Escherichia } \\
\text { coli }\end{array}$ & $\begin{array}{c}\text { Staphococcus } \\
\text { aureus }\end{array}$ \\
\hline \hline 2 & \pm & - \\
\hline \hline 4 & + & + \\
\hline \hline 6 & \pm & - \\
\hline \hline 8 & + & + \\
\hline \hline 10 & + & - \\
\hline
\end{tabular}

Note:(-): No inhibition, $( \pm)=6-9 \mathrm{~mm}$, $(+)=10-14 \mathrm{~mm},(++): 15-22 \mathrm{~mm}$.

Conclusion: 1-For Escherichia coli (G-), compounds $(2,6)$ showed moderate effect on this bacteria, while compounds $(4,8,10)$ showed high activity againstthis bacteria.2-For Staphylococcus aureus $\left(\mathrm{G}^{+}\right)$, compounds $(4,8)$ have highe effect on this bacteria except compounds $(2,6,10)$.

\section{Conclusion}

In conclusion, we describe an efficientprocedure for the chalcones can be synthesized in good yields from aromatic aldehydes and ketones using the catalytic system $\mathrm{NaOH} / \mathrm{EtOH}$. Thus, the present method constitutes a novel synthesis of chalcones with the condition and good yields. The synthesized compounds were characterized by.

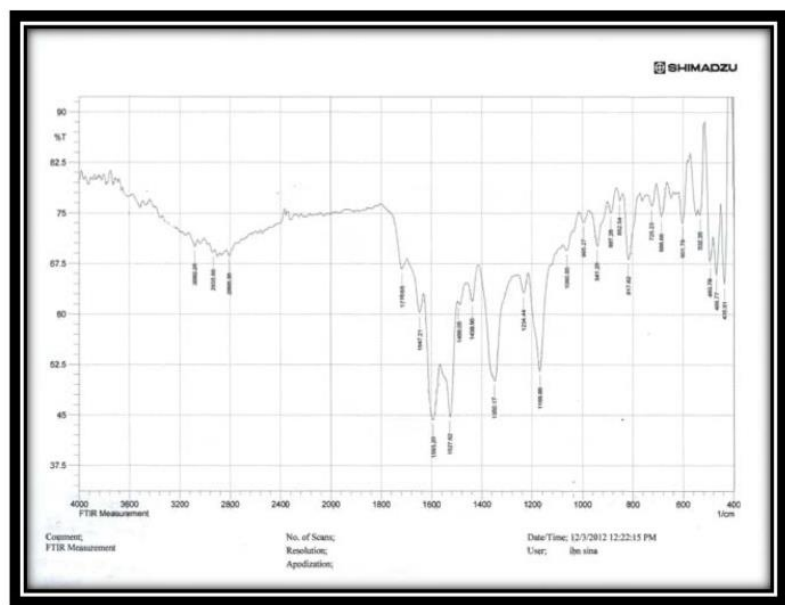

Fig.(1) FT-IR spectrum of compound (1).

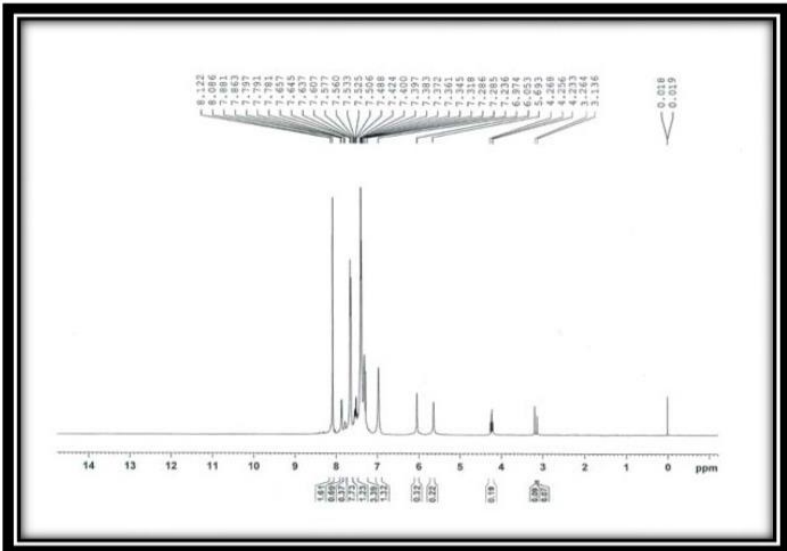

Fig.(2) H-NMR spectrum of compound (1).

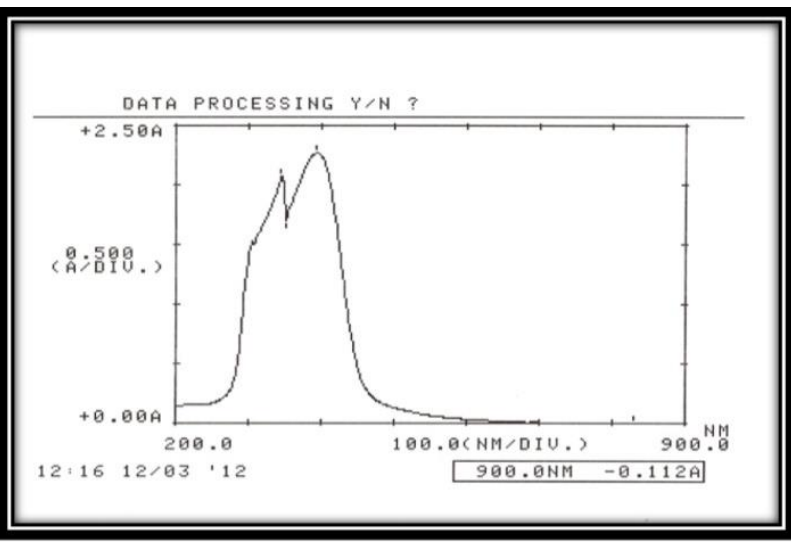

Fig.(3) UV/VIS spectrum of compound (1).

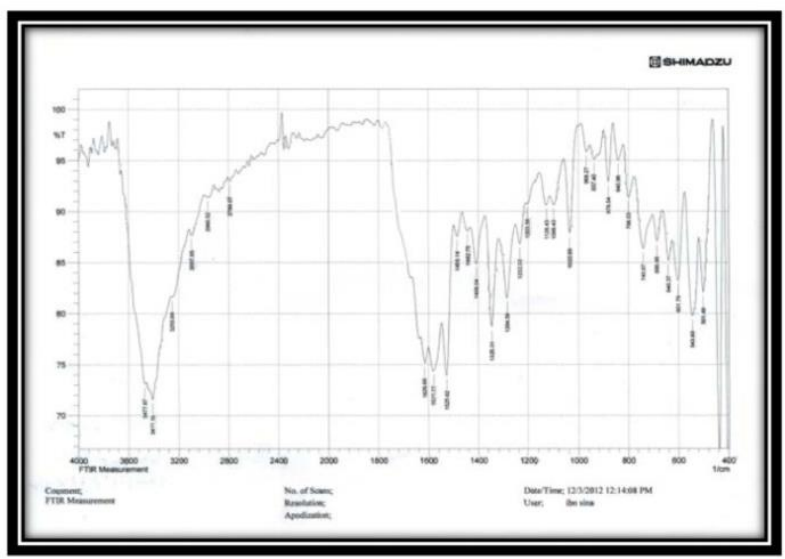

Fig.(4) FT-IR spectrum of compound (10).

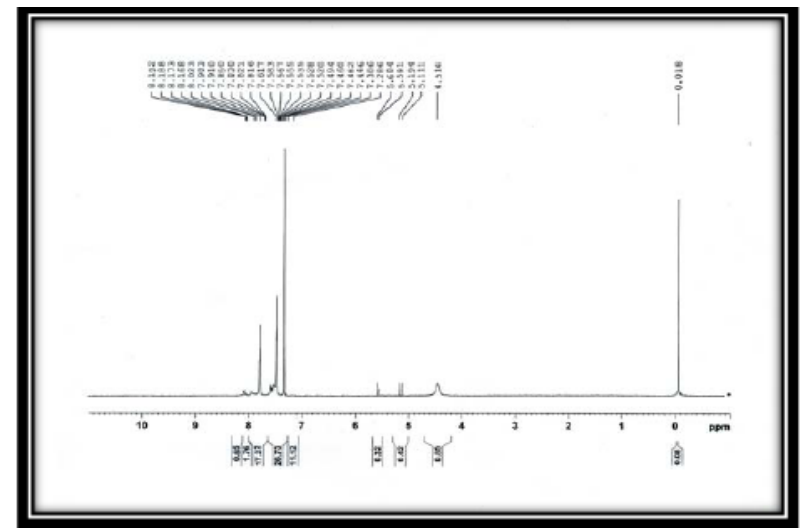

Fig.(5) 1H-NMR spectrum of compound (10). 


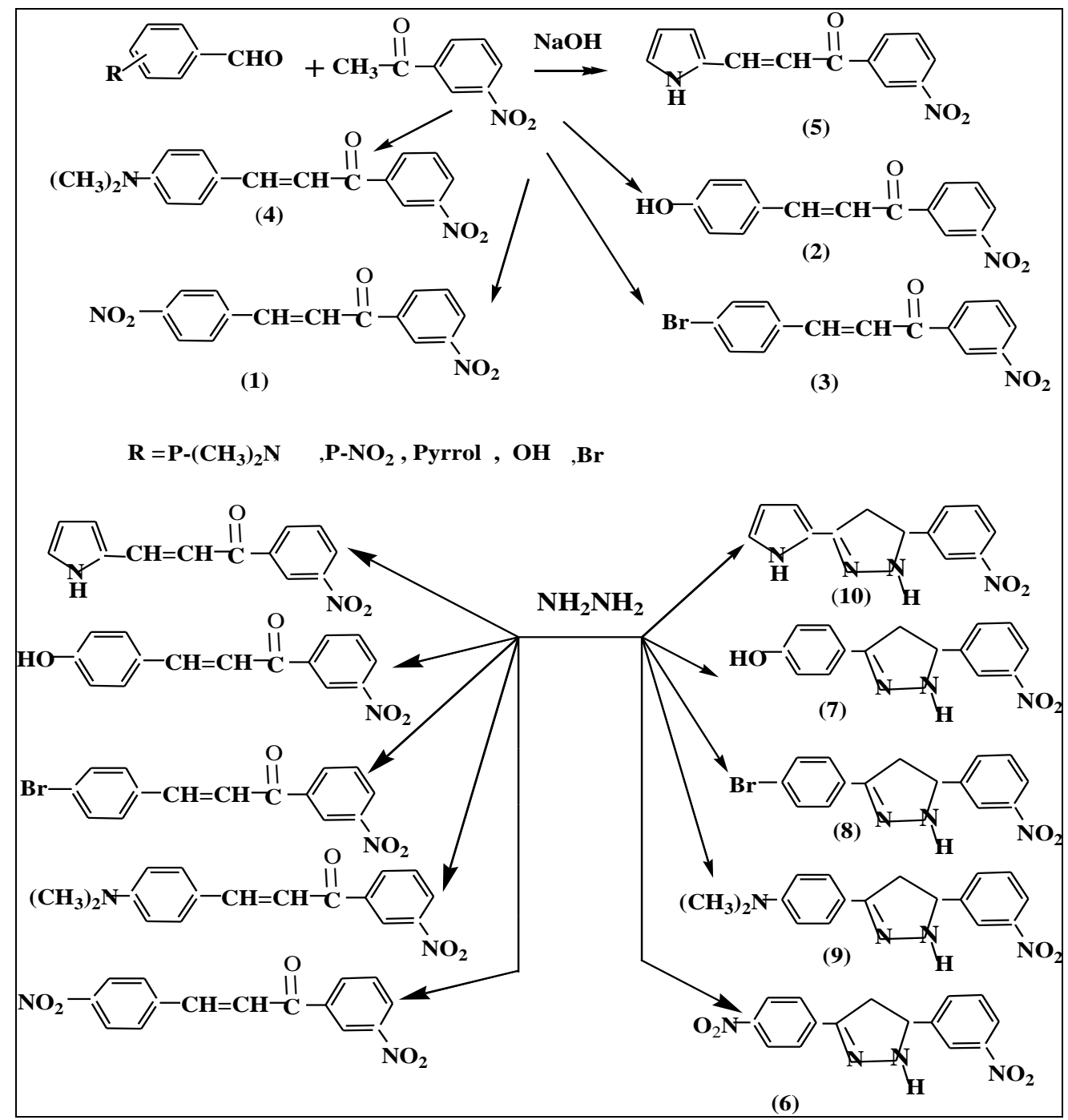

Scheme (1) Represents the prepared compounds.

\section{References}

[1] Nowakowska, Z.; Kedzia, B.; Schroedere, Eur. J. "variety synthesis and characterization of some new chalcone derivatives" Med. Chem. 43, pp:707-713, 2008.

[2] Narender, T.; Papi Redy, K.A. "Asimple and highly efficient method for the synthesis of chalcones by using borontrifluoride-etherate" Tetrahedron Lett.; 48:pp. 3177-3180, 2007.

[3] Mishra, N.; Arora, P.; Kumar, B.; Mishra, L.C.; Bhattacharya, A.; Awasthi, S.K.; Bhasin, V.K. "Synthesis of novel substituted 1,3-diaryl propenone derivatives and their antimalarial activityin vitro". Eur. J. Med. Chem. 43, pp 1530 1535, 2008.

[4] Li, J.T.; Yang, W.Z.; Wang, S.X.; Li, S.H.; Li, T.S." Improved synthesis of chalcones under ultrasoundIrradiation". Ultrason. Sonochem; 9, pp 237-239, 2002.

[5] Karthikeyan, M.S.; Holla, B.S.; Kumari, N.S "Synthesis and antimicrobial studies on novel chloro-fluorine containing hydroxy pyrazolines" Eur. J. Med. Chem.; 42, pp 30-36, 2007.

[6] Rateb N. M., "Convenient synthesis and antimicrobial evaluation of multicyclic Thieno pyridines, Sulfur Silicon" Relat .Elem, 182: pp 2393-2407, 2007.

[7] Dhar D.N, John Wiley \& Sons, "Antiinflammatory and Anticancer effect of Zyflamend against pancreatic cancer "New York, 29(2): pp 107-160, 1981.

[8] M.L.Go, X.Wu, X.L.Liu, "Chalcones: An Update on Cytotoxic and Chemoprotective Properties" Curr. Med. Chem; 12, pp 483, 2005. 
[9] Azarifar D and Ghasemn H, "Paper and board intended to come into contact with foodstuffs. Determination of the fastness of fluorescent whitened paper and board"; molecules 8, pp 642-648, 2003.

[10] H. Hanna, Z.Juncheng, O. Zude, B.Zhou, L.Meiying and Y.Liu, Wuhan University, J. of Natural Science, 15, pp 17177, 2010.

[11]N. C. Oforka, V.N. Mkaenie J. Chemistry, 25; pp.869, 2003.

[12] Jayapal M.R and Sreedhar N.Y "Anhydrous $\mathrm{K}_{2} \mathrm{CO}_{3}$ as catalyst for the synthesis of chalcones under Microwave Irradiation", International Journal of current pharmaceutical research, 2, (10):pp. 975-1491, 2010.

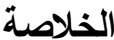

تم تحضير مركبات جديدة من (ז،اسب) ب-

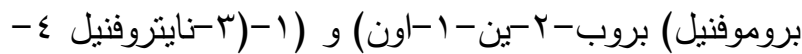

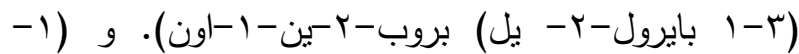

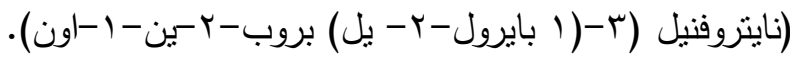

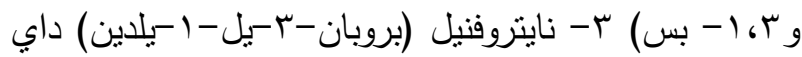

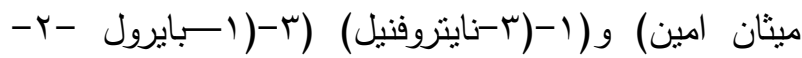

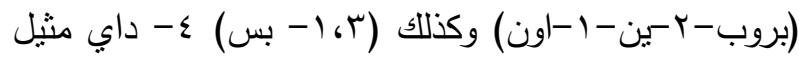

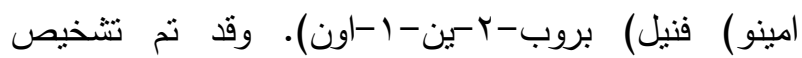

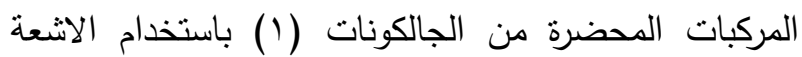
تحت الحمراء والاثعة فوق البنفسجية وطيف الرنين النووي المغناطيسي للبروتون بجانب تحديد درجات الانصهار وتحديد نقاوة المركبات ودراسة الفعالية البايولوجية. 